\title{
Closed Expression for Characteristic Function of CEPE Distribution
}

\author{
Tibor K. Pogány \\ Department of Sciences, Faculty of Maritime Studies \\ University of Rijeka, 51000 Rijeka, Studentska 2, Croatia \\ Tel: 385-51-32-12-40 E-mail: poganj@pfri.hr
}

\begin{abstract}
The recent paper by Maturi and Elsayigh [The Correlation between Variate-Values and Ranks in Samples from Complete Fourth Power Exponential Distribution, Journal of Mathematics Research 1 (2009), No. 1, 14-18] contains an expression for the chatacteristic function of CFPE distribution in the $\$ 2$ as double sum (one infinite) of terms involving special functions. Here, I would like to point that this formula turns out to be a corollary of closed form expression for the characteristic function of so-called Complete Eventh Power Exponential (CEPE) distribution.
\end{abstract}

Keywords: Complete Fourth Power Exponential distribution, Complete Eventh Power Exponential distribution, FoxWright generalized hypergeometric ${ }_{p} \Psi_{q}$ function, Wright's hypergeometric $\phi$ function

The recent paper by Maturi and Elsayigh [The Correlation between Variate-Values and Ranks in Samples from Complete Fourth Power Exponential Distribution, Journal of Mathematics Research 1 (2009), No. 1, 14-18] derived the correlation between variate-values and ranks in a sample from the distribution referred as Complete Fourth Power Exponential (CFPE). The paper contained an expression for the chatacteristic function of CFPE distribution in the $\$ 2$ as double sum (one infinite) of terms involving special functions. Here, I would like to reduce this formula to an explicit and closed form via Wright's hypergeometric type $\phi(\alpha, \beta ; x)$ function. Moreover, this result turns out to be a corollary of closed form expression for the characteristic function of so-called Complete Eventh Power Exponential (CEPE) distribution such that one expresses in terms of Fox-Wright generalized hypergeometric ${ }_{p} \Psi_{q}$ function.

Let us given a r.v. $\xi_{k}$, on a standard probability space $(\Omega, \mathfrak{F}, \mathrm{P})$, with the density function

$$
f_{k}(\alpha, \beta ; x)=\frac{k}{\beta \Gamma(1 /(2 k))} \exp \left\{-\left(\frac{x-\alpha}{\beta}\right)^{2 k}\right\} \quad k \in \mathbb{N}, \alpha \in \mathbb{R}, \beta>0, x \in \mathbb{R} .
$$

Such distribution we will call Complete Eventh Power Exponential (CEPE). Note that $\xi_{1}$ has standard normal $\mathcal{N}\left(\alpha, \beta^{2} / 2\right)$, while $\xi_{2}$ has Complete Fourth Power Exponential (CFPE) distribution considered by Maturi \& Elsayigh (2009) it this journal. They recall (by other origins) the following formula for the characteristic function:

$$
\varphi_{2}(t)=\mathrm{E} \exp \left\{\mathrm{i} t \xi_{2}\right\}=\sum_{m=0}^{\infty} \sum_{j=0}^{\left[\frac{m}{2}\right]}\left(\begin{array}{l}
m \\
2 j
\end{array}\right) \alpha^{m-2 j} \beta^{2 j} \frac{\Gamma(j / 2+1 / 4)}{\Gamma(1 / 4)} \frac{(\mathrm{i} t)^{m}}{m !} .
$$

Here $[x]$ stands for the integer part of some real $x$.

Now, we will derive a more general result. To do this, we give instead of infinite sum a closed form expression for $\varphi_{2}(t)$. In this goal let us introduce the Fox-Wrigth generalized hypergeometric function ${ }_{p} \Psi_{q}(\cdot)$ with $p$ numerator and $q$ denominator parameters, defined by series (cf., e.g., Pogány et al. (2009; Eq. (9.8)):

$$
{ }_{p} \Psi_{q}\left[\begin{array}{c}
\left(\alpha_{1}, A_{1}\right), \cdots,\left(\alpha_{p}, A_{p}\right) \\
\left(\beta_{1}, B_{1}\right), \cdots,\left(\beta_{q}, B_{q}\right)
\end{array} ; z\right]=\sum_{n=0}^{\infty} \frac{\prod_{j=1}^{p} \Gamma\left(\alpha_{j}+A_{j} n\right)}{\prod_{j=1}^{q} \Gamma\left(\beta_{j}+B_{j} n\right)} \frac{z^{n}}{n !}
$$

such that converges for $A_{j}, B_{k}>0,1+\sum_{j=1}^{q} B_{j}-\sum_{j=1}^{p} A_{j}>0$. Let us mention that the confluent case

$$
{ }_{0} \Psi_{1}\left[\begin{array}{c}
- \\
(\beta, B)
\end{array} ; z\right]=\phi(\beta ; B ; z)=\sum_{n=0}^{\infty} \frac{1}{\Gamma(\beta+B n)} \frac{z^{n}}{n !} \quad \beta \in \mathbb{C}, B>0
$$

one calles Wright's function with lower parameters $\beta, B$, compare Gorenflo et al. (1999).

Theorem: Let r.v. $\xi_{k}$ has CEPE distribution, $k \in \mathbb{N}$. Then the characteristic function

$$
\varphi_{k}(t)=\mathrm{E} \exp \left\{\mathrm{i} t \xi_{k}\right\}=\frac{\sqrt{\pi} \exp \{\mathrm{it \alpha}\}}{\Gamma(1 /(2 k))} \cdot{ }_{1} \Psi_{1}\left[\begin{array}{c}
(1 /(2 k), 1 / k) \\
(1 / 2,1)
\end{array} ;-\frac{(\beta t)^{2}}{4}\right] .
$$

Proof: By direct calculation we have

$$
\varphi_{k}(t)=\mathrm{E} \exp \left\{\mathrm{i} t \xi_{k}\right\}=\int_{\mathbb{R}} \mathrm{e}^{\mathrm{i} x t} f_{k}(\alpha, \beta ; x) \mathrm{d} x=\frac{k \exp \{\mathrm{i} t \alpha\}}{\Gamma(1 /(2 k))} \int_{\mathbb{R}} \exp \left\{\mathrm{i} y \beta t-y^{2 k}\right\} \mathrm{d} y .
$$


Expanding the Fourier-kernel exp\{iyt $\}$ into Maclaurin series and making use of the legimite intechange of sum and the integral we conclude

$$
\varphi_{k}(t)=\frac{k \exp \{\mathrm{i} t \alpha\}}{\Gamma(1 /(2 k))} \sum_{m=0}^{\infty} \frac{(\mathrm{i} \beta t)^{m}}{m !} \int_{\mathbb{R}} y^{m} \exp \left\{-y^{2 k}\right\} \mathrm{d} y .
$$

Being the integrand odd function for $m$ odd, all intergals in summands vanish for these values. So, the last expression reduces to

$$
\varphi_{k}(t)=\frac{\exp \{\mathrm{i} t \alpha\}}{\Gamma(1 /(2 k))} \sum_{m=0}^{\infty} \frac{\Gamma(m / k+1 /(2 k))}{\Gamma(2 m+1)}\left[-(\beta t)^{2}\right]^{m} .
$$

Applying now the Legendre duplication formula

$$
\Gamma(2 z)=\frac{2^{2 z-1}}{\sqrt{\pi}} \Gamma(z) \Gamma(z+1 / 2)
$$

to the Gamma-function term $\Gamma(2 m+1)=2 m \Gamma(2 m)$ in the denominator, we clearly deduce

$$
\varphi_{k}(t)=\frac{\sqrt{\pi} \exp \{\mathrm{i} t \alpha\}}{\Gamma(1 /(2 k))} \sum_{m=0}^{\infty} \frac{\Gamma(m / k+1 /(2 k))}{\Gamma(m+1 / 2)} \frac{\left[-(\beta t)^{2} / 4\right]^{m}}{m !}
$$

hence, comparing the last expression with (3), we arrive at (5). The proof is complete.

Corollary: For the r.v. $\xi_{2}$ we have

$$
\varphi_{2}(t)=\frac{\sqrt{2} \pi \exp \{\mathrm{it} \alpha\}}{\Gamma(1 / 4)} \cdot \phi\left(3 / 4 ; 1 / 2 ;-(\beta t)^{2} / 8\right),
$$

where $\phi$ stands for the Wright function definied in (4).

Proof: Specifying $k=2$ in (6) we get

$$
\varphi_{2}(t)=\frac{\sqrt{\pi} \exp \{\mathrm{i} t \alpha\}}{\Gamma(1 / 4)} \sum_{m=0}^{\infty} \frac{\Gamma(m / 2+1 / 4)}{\Gamma(m+1 / 2)} \frac{\left[-(\beta t)^{2} / 4\right]^{m}}{m !} .
$$

Apply once more Legendre's duplication formula to the denominator term $\Gamma(m+1 / 2)$, so the result.

\section{Acknowledgements}

I would like to express my gratitude to the unknown reviewer for useful comments on the earlier version of this article.

The recent investigation was supported in part by Research Project No. 112-2352818-2814 of Ministry of Sciences, Education and Sports of Croatia.

\section{References}

Gorenflo, R., Luchko, Yu. \& Mainardi, F. (1999). Analytical properties and applications of the Wright function. Frac. Calc. Appl. Anal., 2, No. 4, 383-414. [Online] Available: http://www.diogenes.bg/fcaa.

Maturi, T. A. \& Elsayigh, A. (2009). The Correlation between Variate-Values and Ranks in Samples from Complete Fourth Power Exponential Distribution. Journal of Mathematics Research, 1, No. 1, 14-18. [Online] Available: http://ccsenet.org/journal/index.php/jmr/article/viewFile/172/125.

Pogány, T. K., Srivastava, H. M. \& Tomovski, Ž. (2006). Some families of Mathieu a-series and alternating Mathieu a-series. Appl. Math. Comput., 173, No. 1, 69-108. [Online] Available: http://www.sciencedirect.com. 